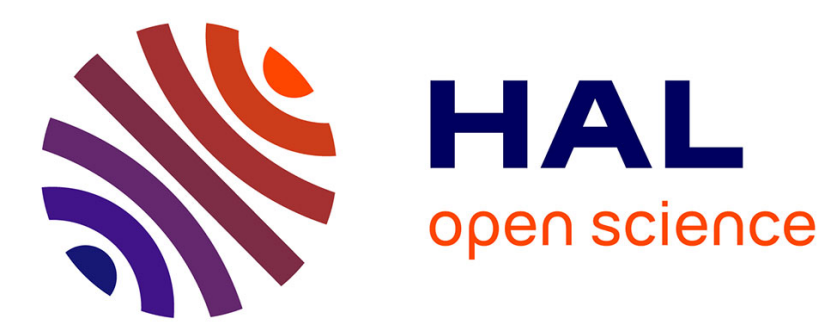

\title{
An Overview of How Semantics and Corrections Can Help Language Learning
}

Dana Angluin, Leonor Becerra-Bonache

\section{To cite this version:}

Dana Angluin, Leonor Becerra-Bonache. An Overview of How Semantics and Corrections Can Help Language Learning. WI-IAT, 2011, France. pp.147-150. hal-00618139

\section{HAL Id: hal-00618139 \\ https://hal.science/hal-00618139}

Submitted on 31 Aug 2011

HAL is a multi-disciplinary open access archive for the deposit and dissemination of scientific research documents, whether they are published or not. The documents may come from teaching and research institutions in France or abroad, or from public or private research centers.
L'archive ouverte pluridisciplinaire HAL, est destinée au dépôt et à la diffusion de documents scientifiques de niveau recherche, publiés ou non, émanant des établissements d'enseignement et de recherche français ou étrangers, des laboratoires publics ou privés. 


\section{An Overview of How Semantics and Corrections Can Help Language Learning}

\author{
Dana Angluin \\ Department of Computer Science \\ Yale University \\ New Haven, USA \\ dana.angluin@yale.edu
}

\author{
Leonor Becerra-Bonache \\ Laboratoire Hubert Curien \\ Université Jean Monnet \\ Saint-Etienne, France \\ leonor.becerra@univ-st-etienne.fr
}

\begin{abstract}
We present an overview of the results obtained with a computational model that takes into account semantics and corrections for language learning. This model is constructed with a learner and a teacher who interact in a sequence of shared situations. The model was tested with limited sublanguages of 10 natural languages in a common domain of situations.
\end{abstract}

Keywords-semantics; corrections; interactive learning

\section{INTRODUCTION}

Can children learn language from the radio? As psychological and linguistic studies have shown [1], interaction with human speakers is crucial for language development. The context in which the utterances are generated also plays an important role in the early stages of children's language acquisition; thanks to the shared context, adult and child can communicate with each other although their grammars are different. Moreover, as some linguistic studies suggest [2], parents often reformulate children's erroneous utterances, to make sure they have understood them. These corrections (in the form of reformulations) are based on the meaning that the child intend to express.

In this paper we present the main features of a computational model that takes into account all these ideas, and we discuss the experimental results obtained with such model. In this model, a teacher and a learner interact in a sequence of shared situations. Both of them engage in comprehension and production of utterances which are intended to be appropriate to their shared situation. Moreover, the teacher is able to correct the learner's utterance, and the learner can detect the intended corrections by the teacher. This setting has allowed us to study the effects of semantics and corrections in language learning.

\section{RELATED WORK}

Most work on computational models of language learning has focused only on language comprehension, and on just a single phenomenon (e.g., single word learning or syntactic category acquisition.) Some of the more empirical works that are focused on comprehension and take into account semantics are those of Siskind [3], Marcken [4], Regier [5] and Bailey [6]. The systems developed by Siskind [7] and Marcken [4] learn word semantics in simulation; it is assumed that the learner already has concepts for each of the words to be learned, and word learning becomes a problem of matching words to concepts. The representation used by these systems is based on compositional semantics. Moreover, Siskind investigated the use of crosssituational analysis to model lexical acquisition. The systems introduced by Regier [5] and Bailey [6] use more realistic simulated data; they focus on learning particular words in isolation, rather than in a linguistic context.

One of the most closely related research efforts is that of Kevin Gold and his collaborators [8], [9]. They introduced a word learning system called TWIG (Transportable Word Intension Generator) that allows a robot to learn compositional meanings for new words. We use a different representation of word meaning, that is not based on Frege's principle of compositionality; this allows us to assign a correct meaning to a sentence even if the sentence is not grammatically correct. Unlike in TWIG, our learner has no initial knowledge of words or grammar, there is no restriction on the number of new words in a sentence given to the learner, and our learner is allowed to interact with the teacher and possibly receive corrections.

Our model is also inspired by Chouinard and Clark's results [2]. They analyze longitudinal data from five children between two and four years old, and show that adults reformulate erroneous child utterances often enough to help learning. Such corrections (in form of reformulations) preserve the intended meaning of the child. Moreover, they show that children not only can detect the corrections, but also make use of them. Our model gives an account of meaning-preserving corrections.

\section{OUR MOdel}

The domain we have used to guide the development of our model is a simplification of the Miniature Language Acquisition task, formalized by Feldman et al. [10]. The task consists of learning a subset of a natural language from sentence-picture pairs that involve geometric shapes with different properties (color, size and position.)

In our model, the input given to the learner consists of a situation and an utterance that denotes something in that situation: 
i) A situation is a collection of objects, properties and relations. These are not intended to be an exhaustive description of the state of the world, but to pick out some aspects of it that are of joint interest to the teacher and the learner. We assume that these objects, properties and relations are recognizable to both the learner and teacher from the outset. A situation is represented as a set of ground atoms over some constants (denoting objects) and predicates (giving properties of the objects and relations between them.) For example, a situation $s_{1}$ consisting of a big red triangle to the left of a big yellow circle is represented by the following set of ground atoms: $s_{1}=\left\{b i 1\left(t_{1}\right), r e 1\left(t_{1}\right)\right.$, $\operatorname{tr1}\left(t_{1}\right)$, le2 $\left(t_{1}, t_{2}\right)$, bi1 $\left(t_{2}\right)$, ye1 $\left.\left(t_{2}\right), \operatorname{ci1}\left(t_{2}\right)\right\}$.

ii) An utterance is a finite sequence of words over a finite alphabet. For example, the star or the star to the right of the purple circle or star of circle small the green. The target language is the set of utterances the teacher may produce in some situation; in our example, this includes utterances like the star or the star to the right of the purple circle but not star of circle small the green. We assume each utterance in the target language is assigned a unique meaning.

The goal of the learner is to learn a grammar for the language that will enable it to produce every appropriate utterance in any given situation. The learner gathers information about the language by engaging in a sequence of interactions with the teacher, where each interaction is related to a new situation. Our model is probabilistic, and what we require is that the probability of learner errors be reduced to very low levels.

The linguistic competence of the teacher is represented by a finite state transducer. This transducer is used by the teacher both to produce utterances appropriate to the given situation, and to analyze the utterances of the learner.

The learner and teacher share the same description of the situation. They also know a shared set of categories that classify a subset of the predicates into similarity groups. For example, in our domain we have three different categories: one for shape predicates, one for color predicates, and one for size predicates. No category contains the positional relations. The categories facilitate generalization by the learner, and are used by the teacher in analyzing incorrect utterances of the learner.

Figure 1 summarizes one interaction between learner and teacher. More details about our model and a description of the algorithms used by the learner and teacher to carry out the steps of this process can be found in [11].

\section{Testing on Natural Language SAmples}

We have implemented and tested our model in the example domain of geometric shapes with limited sublanguages of ten different natural languages: English, German, Greek, Hebrew, Hungarian, Mandarin, Russian, Spanish, Swedish,
1) A new situation is generated and presented to the learner and teacher.

2) The learner uses its current grammar to attempt to produce an utterance.

3) The teacher analyzes the learner's utterance (if any) in the context of the situation.

4) If the learner's utterance has an error in form or an error in meaning, the teacher decides randomly (using the correction probability) whether to correct the learner.

5) The teacher produces an utterance appropriate to the situation.

6) The learner analyzes the teacher's utterance and updates its current grammar.

Figure 1. Summary of one interaction in the learning cycle.

and Turkish. These experiments have allowed us to explore questions about the role of semantics and corrections in language learning.

In our experiments, each situation has two objects, each with three attributes (shape, color and size), and one binary relation between the two objects (above or to the left of.) The attribute of shape has six possible values (circle, square, triangle, star, ellipse, and hexagon), that of color has six possible values (red, orange, yellow, green, blue, and purple), and that of size three possible values (big, medium, and small.) There are 108 distinct objects and 23,328 distinct situations. Situations are generated uniformly at random.

The utterances in a situation are phrases intended to denote one of the objects, for example, the circle, the orange star, or the small purple hexagon below the medium green square. There are 168 meanings referring to a single object and 112,896 meanings referring to two objects, for a total of 113,064 possible meanings.

In each of these languages, the 113,064 possible meanings are instances of 68 general forms: 4 referring to a single object and 64 referring to two objects. For English, examples of general forms are $\left(\operatorname{shape} 1\left(x_{1}\right)\right),\left(\operatorname{color} 1\left(x_{1}\right)\right.$, shape $\left.1\left(x_{1}\right)\right)$ and $\left(\operatorname{size} 1\left(x_{1}\right)\right.$, shape $1\left(x_{1}\right)$, le2 $\left(x_{2}, x_{1}\right)$, shape1 $\left.\left(x_{2}\right)\right)$. We refer to these languages as the 68-form languages.

For each language we consider, we consulted at least one speaker of the language to help us construct a meaning transducer to translate appropriate phrases in the language to all 113,064 possible meanings. Each transducer was constructed to have exactly one accepted phrase for each possible meaning.

To help understand the effect of different aspects of the learning problem, we also considered reduced sublanguages, consisting of the utterances that refer to a single object (168 utterances) and those that refer to two objects, but include all three attributes of both (46,656 utterances.) Thus, for English the utterances in the reduced sublanguage include the star, the blue triangle and the medium purple ellipse 
to the left of the medium red square but not the circle below the yellow hexagon. Each meaning in the reduced sublanguage is an instance of one of 8 general forms, but most of the lexical and syntactic complexity of the language is preserved. We refer to these reduced sublanguages as the

\section{8-form languages.}

The level of performance of a learner attempting to learn a given language $L$ is measured using two quantities: the correctness and completeness of the learner's utterances in a given situation.

i) Correctness: sum of the probabilities of the learner's utterances that are in the correct denoting set.

ii) Completeness: fraction of the correct denoting utterances that appear in the set of learner utterances.

The averages of correctness and completeness of the learner in 200 randomly generated situations is used to estimate the overall correctness and completeness of the learner. We say that a learner reaches a level $p$ of performance if both correctness and completeness are at least $p$.

We conducted two different sets of experiments. In all of them, we set a target level of performance of $p=0.99$. The first set of experiments tries to answer the following question: What are the effects of a correcting and a noncorrecting teacher for the learner to accomplish the learning task? The second set of experiments tries to see whether the presence of semantics facilitate the learning process. We review the results obtained in the next sections. More details can be found in [11] and [12].

\section{A. What are the effects of corrections?}

In order to see the effects of corrections in language learning, we have compared the number of interactions needed by the learner to accomplish the learning task to a high level of performance in two different situations: i) The teacher does not correct the errors produced by the learner; ii) The teacher corrects the incorrect learner utterances.

First of all, we tested the number of interactions needed by the learner to reach the 0.99 level of performance, for each 68-form language, with correction probabilities of 0.0 and 1.0 .

We obtained the following results. When the teacher does not correct the learner (i.e., correction probability is 0.0 ), we could distinguish two different groups: Greek and Russian, each with at least 3400 interactions and the rest of the languages, each with at most 1000 interactions. The first observation is that the learner achieves correctness and completeness of 0.99 for each of these languages after being exposed to a small fraction of all possible situations and utterances. Even the maximum number of interactions needed for Russian (concretely, 3700 interactions) involve at most $16.5 \%$ of all possible situations and at most $3.5 \%$ of all possible utterances by the teacher.

When the teacher corrects the learner (i.e., correction probability is 1.0), we see the same two groups of languages:
Greek and Russian versus the rest of the languages tested. For Greek and Russian, there is a clear decrease in the number of interactions required to achieve level of performance 0.99; for Greek, a decrease of about $24 \%$, and for Russian a decrease of about $21 \%$. However, the languages in the other group show no clear effect of corrections in these trials. The question is: Why?

It is important to note that in these trials the learner processes a teacher utterance in exactly the same way regardless of whether the learner classifies it as a correction. That is, the learner does not do anything special for perceived corrections. Thus, the improvements in performance for Greek and Russian depend entirely on the difference in behavior of the teacher. In particular, the non-correcting teacher and the correcting teacher produce different distributions of utterances, and this change in the learners environment changes the performance of the learner. This suggests that studies of correction in natural languages should consider the possibility that corrections may affect the learning process even when the learner cannot detect corrections (or does not attempt to.)

If we further examine the results, we can see that for the group consisting of Greek and Russian, the learner had acquired its last general form in attaining performance level 0.90 , and then, the attainment of the 0.99 level of performance is limited by the need to improve the correctness of the phrase choices. However, for the group consisting of the other languages, the bottleneck is the acquisition of all 68 correct general forms. The teacher's corrections tend not to help with the acquisition of new general forms.

In order to see if correction would have an effect when the bottleneck of acquiring general forms was removed, we conducted the same experiments with the 8-form languages. The results obtained with these reduced sublanguages are the following. We can see an improvement of over $30 \%$ in the number of interactions to reach performance level 0.99 in going from correction probability 0.0 to correction probability 1.0 . The intermediate correction probabilities give intermediate values. The corrected learners seem to be slightly slower to reach the 0.60 level of performance. The data on numbers of general forms for these runs show that learners had overwhelmingly acquired their last general form by the time they reached the 0.90 level of performance, where the superior performance of the corrected learners becomes very evident.

These experiments confirm our hypothesis that corrections can substantially help the learner when the problem of acquiring all the general forms is not the bottleneck.

\section{B. What are the effects of semantics?}

Our second set of experiments was focused on investigating whether the presence of semantics facilitates the learning process. Unlike the previous experiments, we only consider a non-correcting teacher. Therefore, the teacher 
simply produces a randomly drawn denoting utterance for the situation, independent of the learner's production. Note that because we consider only the case of a non-correcting teacher, only positive data is provided to our learner.

In order to answer this question, we compared the performance of our system to the ALERGIA algorithm. ALERGIA is one of the most successful approaches to the problem of learning PDFA (probabilistic deterministic finite automata) from a positive sample, and it has become the basis of comparisons for algorithms that have been subsequently proposed. A full description of this algorithm can be found in [13].

We used the ALERGIA algorithm to learn limited sublanguages of Spanish and English, specifically, the 68form sublanguages of Spanish and English. We compared the number of examples needed to reach a high level of performance by the ALERGIA algorithm with the number of interactions needed by our system. The utterances given to ALERGIA were generated in the same way as the utterances of a non-correcting teacher, i.e., we generated a sequence of $n$ pairs of a random situation and a randomly chosen denoting utterance, and extracted the sequence of utterances to form the input to ALERGIA.

We ran the ALERGIA algorithm to estimate the minimum number of utterances required to acquire these two languages, and the results obtained were the following. In the case of Spanish, instead of 1000 situation/utterance pairs, ALERGIA needs 6000 utterances. For English, the difference is still larger; instead of 700 situation/utterance pairs, ALERGIA needs 15000 utterances. These results show that the number of examples is drastically reduced when semantics is taken into account, suggesting that the presence of semantics signicantly facilitates the learning process, even when the teacher is non-correcting. Our results in the previous section suggest that if in addition to semantics, corrections are taken into account (e.g., the teacher's correction probability is set to 1.0), the improvement for some learning tasks can be even greater.

\section{CONCLUSION AND FUtuRE WORK}

Our model and results show that: i) Semantics can significantly reduce the number of examples needed to acquire limited sublanguages of natural language; ii) A teacher can offer corrections to the learner, the learner can detect them, and that such corrections can significantly reduce the number of interactions needed by the learner to accomplish the learning task (to a high level of performance), even if the learner does not treat corrections specially.

A future direction of research is to improve our current model of language; it is very simplified, and there are many issues it does not deal with properly (e.g., morphological relations between words, phonological rules for word choice, polysemous words, etc.) It would also be interesting to see if a learner can use the ability to detect corrections to accelerate the learning process.

\section{ACKNOWLEDGMENT}

The work of the first author was supported by the National Science Foundation under Grant CCF-0916389. The second author thanks the support given by the Network of Excellence PASCAL 2.

\section{REFERENCES}

[1] S. Ervin-Tripp, "Some strategies for the first two years," in Cognitive development and the acquisition of language. Academic Press, 1973.

[2] M. Chouinard and E. Clark, "Adult reformulations of child errors as negative evidence," Journal of Child Language, vol. 30, pp. 637-669, 2003.

[3] J. Siskind, "A computational study of cross-situational techniques for learning word-to-meaning mappings," Cognition, vol. 61, pp. 39-61, 1996.

[4] C. de Marcken, "Unsupervised language acquisition," Ph.D. dissertation, MIT, 1996.

[5] T. Regier, The Human Semantic Potential: Spatial Language and Constrained Connectionism. Cambridge, MA: MIT Press, 1996.

[6] D. Bailey, "A computational model of the role of motor control in the acquisition of action verbs," Ph.D. dissertation, U.C. Berkeley, 1997.

[7] J. Siskind, "Lexical acquisition in the presence of noise and homonymy," in Proceedings of the 12th National Conference on Artificial Intelligence (AAAI 94). MIT Press, 1994.

[8] K. Gold, "Using sentence context and implicit contrast to learn sensor-grounded meanings for relational and deictic words: The TWIG system," Ph.D. dissertation, Yale University, 2008.

[9] K. Gold, M. Doniec, C. Crick, and B. Scassellati, "Robotic vocabulary building using extension inference and implicit contrast," Artifical Intelligence, vol. 173 (1), pp. 145-166, 2004.

[10] J. A. Feldman, G. Lakoff, A. Stolcke, and S. Weber, "Miniature language acquisition: A touchstone for cognitive science," in Proceedings of the 12th Annual Conference of the Cognitive Science Society. MIT, Cambridge, MA, 1994, pp. 686-693.

[11] D. Angluin and L. Becerra-Bonache, "A model of semantics and corrections in language learning," Computer Science Department, Yale University, Tech. Rep. YALE/DCS/TR1425, 2010.

[12] _ _ "Effects of meaning-preserving corrections on language learning," in CoNLL 2011: 15th Conference on Computational Natural Language Learning. In press.

[13] R. Carrasco and J. Oncina, "Learning stochastic regular grammars by means of a state merging method," in Proc. of ICGI 94: 2nd Int. Colloquium on Grammatical Inference, 1994, pp. 139-152. 